\title{
A delayed death due to manual strangulation: Is it medical negligence?
}

\author{
Lakmali MGN ${ }^{1}$, Warushahennadi $\mathrm{J}^{2 *}$, Mahinda $\mathrm{HA}^{3}$
}

\section{Introduction}

Manual strangulation is a mode of asphyxia death produced by pressure of the hands, forearm or other limb against the neck, compressing the internal structures. [1] The combined effects of air way occlusion and disturbed cerebral vascular supply and drainage are responsible for the lethal insult in most deaths from strangulation [2] and it accounts for a reasonable number of violent deaths but some people may survive. The survivors are most often victims of intimate partner violence and sexual assaults. [3] Sometimes, strangulation may cause delayed deaths. The causes for delayed deaths are mainly due to pulmonary complications like adult respiratory distress syndrome, aspiration pneumonia or pneumonitis and carotid artery dissection causing vascular compromise. [4] In addition to the above mentioned causes the delayed upper air way oedema, haematoma, vocal cord immobility, laryngeal fractures and neck abscess formation all may contribute to an unstable air way in survivors following strangulation. [5]

This is a delayed death of a victim following manual strangulation in which the victim died due to a largepyothorax following oesophageal necrosis. The aim of this case report is to demonstrate the importance of detailed clinical assessment in victims of manual strangulation, to prevent the occurrence of delayed deaths due to air way collapse. This is an extremely rare complication of manual strangulation which leads to the death of a victim several days after the attempted manual strangulation by the boyfriend.

${ }^{1}$ Postgraduate Trainee, JMO's office, Teaching Hospital Karapitiya, Sri Lanka, ${ }^{2,3}$ Senior Lecturer, Department of Forensic Medicine, Faculty of Medicine, Karapitiya, Sri Lanka

*Corresponding author: Warushahennadi J, Tele: 0094773909525, e-mail address: anakiwh@gmail.com

\section{Case Report}

A 36 year old mother of 3 children was admitted to a local hospital following an assault and attempted manual strangulation by her boyfriend. She had developed dysphagia and was transferred to a teaching hospital four days later for specialized management. On admission to the teaching hospital there were no external neck injuries and enlarged right submandibular lymph nodes were noted. She had been referred to the consultant ear nose throat Surgeon. The laryngoscopy revealed vocal code haematoma and was treated with intra venous antibiotics for three days and was discharged from the teaching hospital on the fourth day. Three days after been discharged from the hospital, the dysphagia became worse and she was found dead on admission to the hospital.

A medico legal autopsy was performed on the order of the Magistrate. There were no external injuries on the body or on the neck. The neck dissection was performed using the Prinslo and Gordon technique. There were circular and oval shaped contusions sizes varying from $1 \mathrm{~cm}$ to $2 \mathrm{~cm}$ in diameter on the either sides of the upper neck on subcutaneous tissues and on playtime (figure 1).

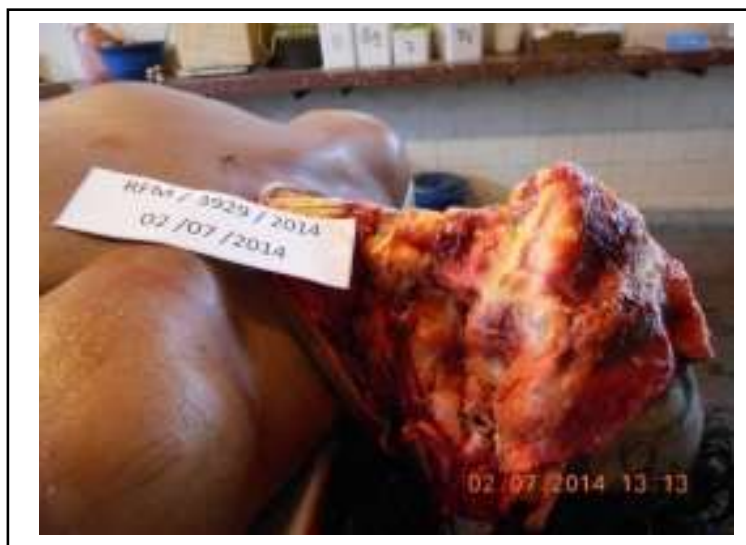

Figure 1: Sub cutaneous contusions on the neck 
Bilateral sub mandibular lymph nodes were enlarged; right side more than the left. There was necrosis of the soft tissues surrounding the pharynx and on upper one third of the oesophagus with an approximately 1 $\mathrm{cm}$ size perforation in the anterior wall of the oesophagus. An abscess formation was observed on the surrounding tissues which extended through the tissue plains to the right chest cavity (figure 2).

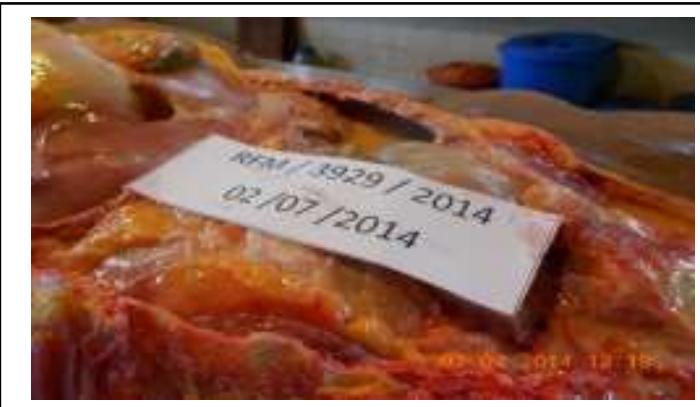

Figure 2: Neck abscess and pus collection in the right pleural space
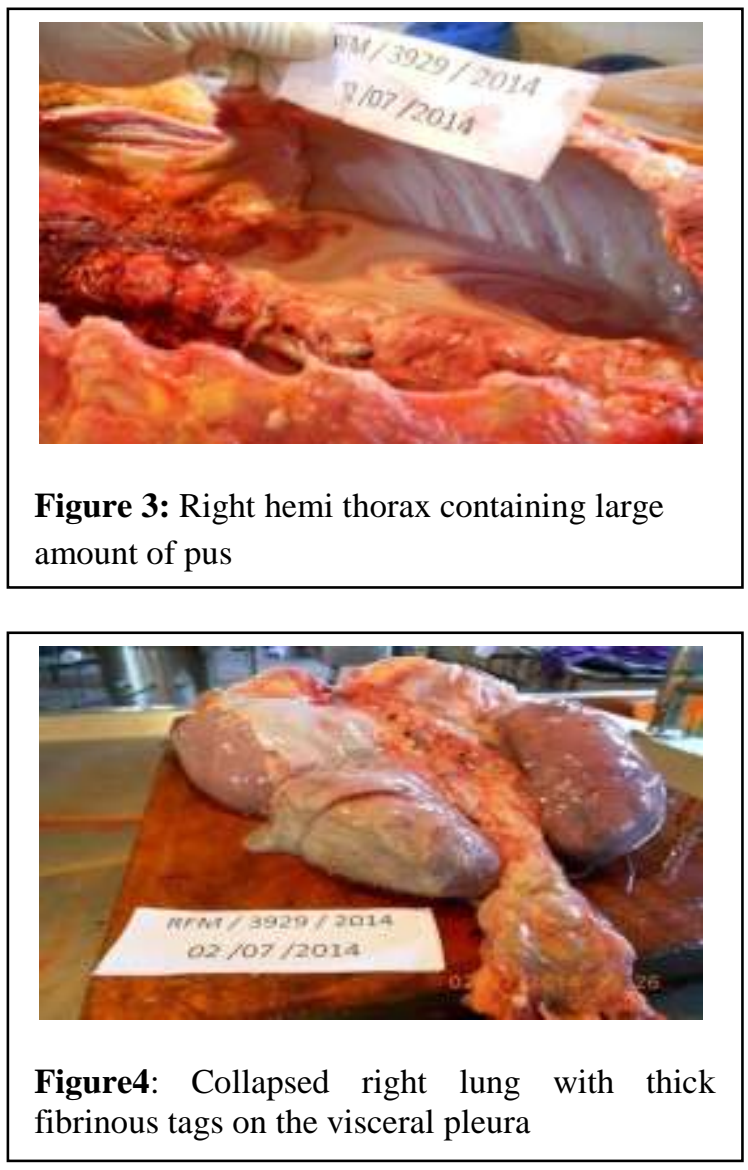

There was right sided massive pyothorax containing $1000 \mathrm{ml}$ of pus with partial collapse of the right lung (figure 3). The right visceral pleura showed fibrinous tag formation (figure 4). The left lung and other organs were free of any injuries or pathological conditions. There were no features of septicemia in the body.

The cause of death was concluded as right sided pyothorax due to oesophageal necrosis and rupture following attempted manual strangulation.

\section{Discussion}

The hallmark of manual strangulation is fingertip bruises and fingernail marks on the neck. [6] But absence of those injuries does not preclude strangulation. In many cases of strangulation there is extensive external injury to the neck, while in others there are few or no external manifestations of trauma. [2] Fingertip bruises are approximately circular or oval. [2]They can produce contusions in underlying tissues such as strap muscles frequently. Absence of fingernail marks on the skin does not imply absence of bruising in the deeper tissues. A single small area of bruising in any muscle of the neck usually on either side of the midline over the thyroid cartilage or hyoid bone may be all that is found in a case of manual strangulation. [2] In this case, there were no external injuries on the neck but circular and oval contusions were found on the subcutaneous tissues and on the platysma which suggests the history of strangulation. Most victims of strangulation may have no visible injuries and the opportunities for higher level criminal prosecution have been missed. [5]Further the contusions on the skin may not be visible on dark skinned people which may be the reason in our case. She died 10 days after the incident of attempted manual strangulation. The contusions on the skin may have healed which may be the other explanation for the absence of contusions on the skin at the autopsy.

Laryngeal cartilage fractures, especially the superior cornu of hyoid bone is common in manual strangulation. [6] But there were no laryngeal cartilage fractures observed at the autopsy. The constriction force may not have been severe enough to cause the fractures on this survived victim. Also the relatively young age of the victim with noncalcified flexible bones and laryngeal cartilages may be another reason for the absence of fractures.

During the strangulation the upper part of oesophagus must have been confused by kinking of bony and cartilaginous structures in the neck. This had caused inflammation of oesophageal tissue and enabled the passage of microbes from the gut to neck tissues which ultimately lead to abscess formation. Pus 
collection had been drained in to the thoracic cavity via the tissue planes and lead to the formation of pyothorax and partial collapse of right lung.

Delayed deaths due to mechanical asphyxia may occur due to anoxic cerebral damage and subsequent hypoxia. But such patients almost always follow periods of unconsciousness. Other potentially lethal sequalae include massive subcutaneous and mediastinal emphysema due to tracheal and laryngeal lacerations or airway occlusion as a result of oedematous or haemorrhagic swelling of pharyngeal tissues or ary-epiglottic folds. [6]However, in this case, the patient was not in a vegetative state. Also there were no demonstrable subcutaneous and mediastinal emphysema. There was no haematoma formation in the neck. So the sole mechanism of death is delayed progressive air way obstruction from the pressure effects of the neck abscess and pyothorax.

A few case reports of delayed deaths due to manual strangulation have been in the literature. In some cases the mechanism of death has not been well established. In 1996 Anscombe and Knight have published a case of attempted strangulation with a survival time of 7 days. There the authors convinced that the cause of death was hypoxic brain damage, although they have not mentioned any gross pathological lesion or any specific histopathological finding. [7] In 1992 Dasari $\mathrm{H}$ et el have reported 2 cases of delayed deaths for 7 and 14 days respectively after attempted hanging. The cause of death was certified as either hypoxic brain damage or a combined effect of cerebral damage due to cerebral ischaemia and bilateral consolidation of lungs. [8] In 2005 Badkur D et el reported a case of attempted manual strangulation who survived19 days and died due to cerebral infarction from occlusion of cerebral vessels. [9]

Although the patient was under specialized care for 4 days, the clinicians have failed to diagnose the cause for the dysphagia. Did the doctors fail to give a reasonable duty of care towards this patient? Reasonable duty of care in this clinical situation includes obtaining X-ray of the neck, laryngoscopy and upper gastrointestinal endoscopy. [1]Despite the persistent dysphagia upper gastrointestinal endoscopy had not been performed. Therefore, one can argue that the doctor had failed to give a reasonable duty of care thereby the patient died of the complication of attempted manual strangulation. But this is a very rare presentation and no previous case has been reported. The overall management of the patients with dysphagia includes early imaging studies, endoscopic assessment, and continued airway monitoring in an intensive care unit [4] which was done on this patient while in the ward. Therefore can it be a medical negligence or is it just a mishap?

In conclusion, victims with attempted manual strangulation can survive despite the internal neck injuries which can lead to delayed fatal airway collapse. Proper investigations for the complications and proper treatments could prevent such deaths.

\section{References}

1. Funk M, Schuppel J. Strangulation injuries.Wisconsin Medical Journal 2003.102(3), 41-45.

2. Adelson L. The Pathology of homicide. A vade mecum for pathologist, prosecutor and defence counsel. Charles C Thomas publisher: Springfield, Illinois; 1974.

3. Hawley DA. Forensic Medical Findings in Fatal and Non-fatal Intimate Partner Strangulation Assaults. 2012; dhss.alaska.gov

4. Kuriloff DB, PincusRL. Delayed airway obstruction and neck abscess following manual strangulation injury.1989 Oct; 98(10):824-7.

5. Strack GB, McClane GE, Hawley D. A review of 300 attempted strangulation cases part $\mathrm{i}$ : criminal legal issues. Journal of Emergency Medicine 2001 Oct; 21(3), 303309.

6. Spitz WU, Spitz DJ. Spitz \& Fisher's Medico-legal Investigation of Death, Guidelines for the application of Pathology to Crime investigation: $4^{\text {th }}$ Edition. Charles C Thomas, Publisher ltd; Springfield, Illinois. 2006.

7. Anscombh AM, Knight BH. Case report. Delayed death after pressure on the neck, possible causal mechanisms and implications for mode of death in manual strangulation discussed. Forensic Science International. 1996 April 23; 78(3): 193-97.

8. Harish D, Anil K, Sirohiwal BL, Dikshit PC. Delayed death in hanging: case reports. Journal of Forensic Medicine and Toxicology. 1992; 9: 48-50.

9. Badkur DS, Arorae A, Jain CS. Delayed death in a case of attempted strangulation; Mechanism of cerebral thrombosis and infarction. A case report. Journal of Indian Academy of Forensic Medicine. 2005; 27(4). 263265 . 